$$
\begin{array}{r}
666.74 .002 .005=60 \\
633.9
\end{array}
$$

\title{
prensa automática para moldear azulejos
}

\author{
R. S. HARDING y A. N. GILSON
}

El número de tipos de prensas completamente automáticas para el moldeo de azulejos es muy reducido $y$, por tanto, se ha considerado de interés la descripción de una máquina, cuyo principio de funcionamiento es poco corriente.

Según se ve en la fig. 1, es muy compacta, ocupando tan sólo una superficie de $130 \times 167 \mathrm{~cm}$. La mayor parte de los mecanismos se encuentran en la mitad inferior de la máquina. La presión, de unas 40 toneladas, es aplicada desde la parte inferior por medio de un pistón y un juego de excéntricas.

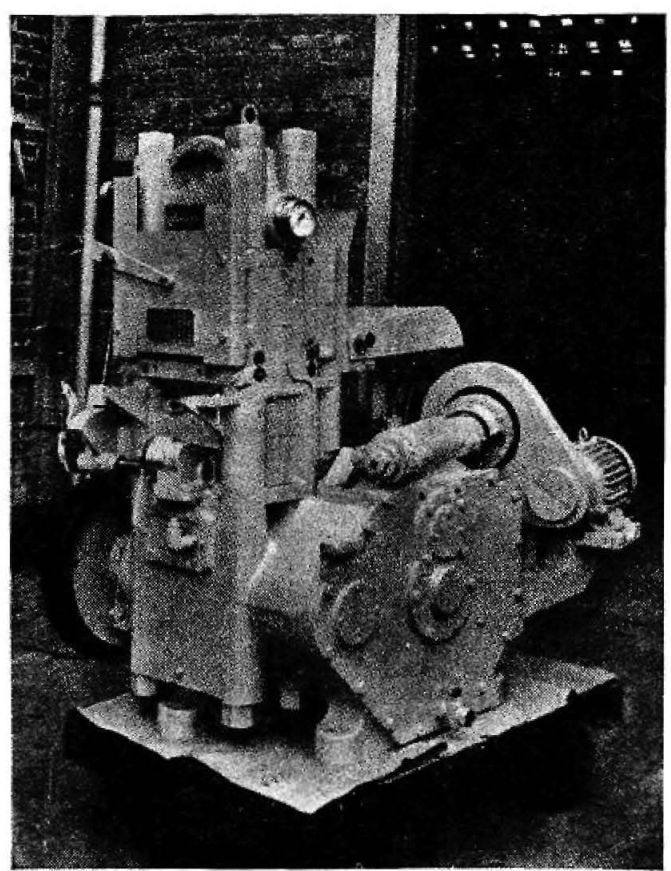

Fi. 1.-Prensa automática para azulejos
La cabeza de la prensa, que está sometida a grandes esfuerzos mecánicos, es muy robusta. Cuatro columnas de acero soportan la parte superior y fijos a ambos lados del conjunto central hay dos cuerpos laterales.

Tiene una tolva de alimentación susceptible de ajuste para conseguir una distribución uniforme de la arcilla en el molde. También puede regularse, para obtener un determinado espesor, aun cuando la prensa esté en funcionamiento.

Se han adoptado ciertas medidas contra los accidentes. El volante y otras partes móviles llevan protecciones. Un dispositivo especial impide poner en marcha la máquina si no tiene colocada la protección frontal. La cinta trapezoidal y el mecanismo de la tolva de alimentación llevan también una adecuada protección.

La prensa corriente va provista de una caja de moldeo de $24,6 \mathrm{~mm}$ que puede producir azulejos de 4 a $9,5 \mathrm{~mm}$ de espesor; dependiendo esto del grado de compactación de la arcilla. Mediante pequeñas modificaciones se pueden moldear azulejos más gruesos, de incluso $12 \mathrm{~mm}$ de espesor, $y$, por el contrario, inferiores a $4 \mathrm{~mm}$ si fuese necesario.

Su capacidad oscila entre 17 y $20 \mathrm{com}$ presiones por minuto y pueden obtenerse las producciones que a continuación se indican, aunque se emplee un amplio margen de tiempo para limpiar el molde.

Azulejos de $100 \times 100 \mathrm{~mm}$ - dos por prensada $-146 \mathrm{~m}^{2}$ cada 8 horas.

Azulejos de $114 \times 114 \mathrm{~mm}$-dos por prensada- $184 \mathrm{~m}^{2}$ cada 8 horas.

Azulejos de $152 \times 76 \mathrm{~mm}$-dos por prensada $-167 \mathrm{~m}^{2}$ cada 8 horas. 
Azulejos de $152 \times 152 \mathrm{~mm}$ - uno por prensada - $167 \mathrm{~m}^{2}$ cada 8 horas.

Azulejos de $200 \times 100 \mathrm{~mm}$ - uno por prensada - $150 \mathrm{~m}^{2}$ cada 8 horas.

Según las necesidades de la fábrica se pueden emplear diferentes tipos de aparatos refrentadores, pero el uso más frecuente es el alisador, proyectado en un principio para utilizarlo con esta máquina (fig. 2)

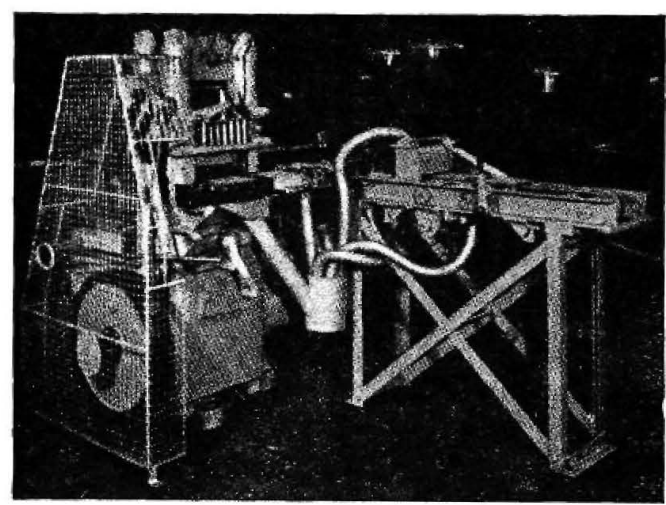

Fig. 2. - Prensa con defensas, alisadora y colector de polvos

que tiene sus movimientos sincronizados con los de la prensa. Los azulejos quedan alisados por arriba y por abajo y cepillados por ambas superficies.

Las dos máquinas, prensa y alisadora, se han construido de tal forma que el polvo que se produce durante el moldeo se ha procurado reducir al mínimo.

\section{Mecanismo de propulsión}

Un motor de 3 CV y 1.800 r.p.m. mueve, por medio de correas trapezoidales, la prensa. La velocidad puede variarse, según los ciclos de prensado que se requieran, por medio de una polea de paso variable, montada sobre el eje del motor. La polea transmite el movimiento, por medio de un embrague, a un eje que lleva acoplado un tornillo sinfín. El sinfín ataca a una rueda dentada, la cual arrastra al árbol de levas de la máquina.

En la figura 3 puede verse la rueda dentada que al mismo tiempo muevellajbomba de aceite sumergida.

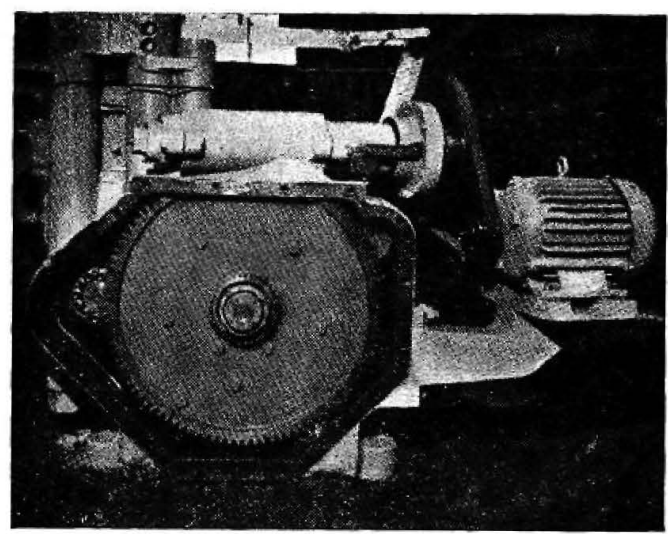

Fig. 3.-Motor, embrague, engranaje y transmisión con cubierta lateral desmontable

El embrague de fricción, que es de tres discos, posee un dispositivo de freno que actúa automáticamente cuando el mando de puesta en marcha se lleva a la posición "of». La prensa puede pararse en la posición más conveniente de la actuación del pistón, 0 , por el contrario, cuando éste se encuentra en el punto más alto del recorrido.

El árbol de levas (fig. 4) es la parte más importante $y$, sin duda alguna, puede llamarse el centro de regulación de todo el funcionamiento automático de la máquina. Este árbol consta de seis levas. Siguiendo un cierto orden de derecha a izquierda, la primera es la leva de la tolva de alimentación; la siguiente, la leva que actúa los pistones superiores de moldeo; las dos que suceden a éstos, las que accionan los moldes inferiores; a continuación, se encuentra la leva que pone en movimiento el dispositivo para expulsar de los moldes los azulejos ya prensados, y la última es la que detiene el movimiento cuando el molde superior está en su posición más elevada. 


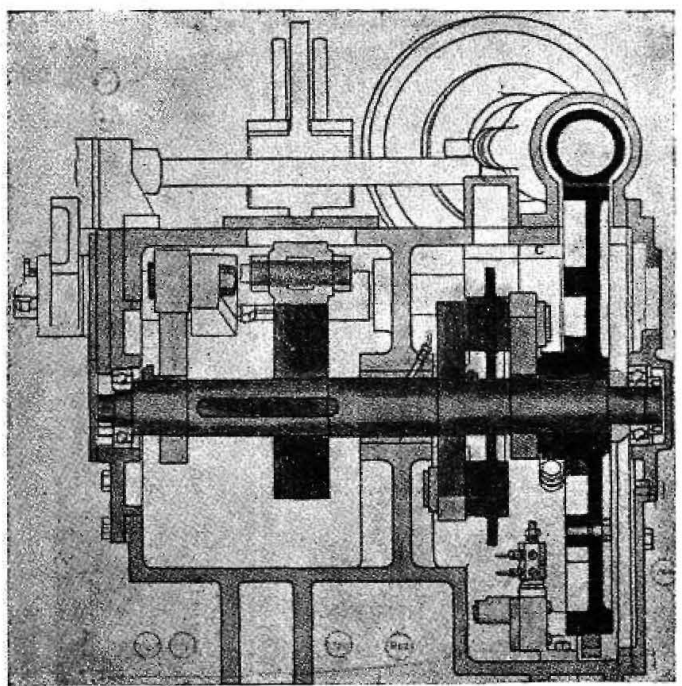

Fig. 4.-Sección transversal de la base de la prensa en la que se puede ver el juego de levas

\section{Mecanismo de alimentación}

En la leva de la tolva de alimentación actúa un brazo, que tiene el alimentador, comunicando un movimiento alternativo a la tolva. Para evitar los daños que pueden producir las materias extrañas contenidas por las tierras, hay previsto un dispositivo de seguridad. La cantidad de tierra que suministra a los moldes la tolva de alimentación puede variarse, de acuerdo con la clase de azulejo que se desea producir, su espesor, grado de compresión y clase de tierra utilizada.

Como puede verse en la figura 5 , la tolva, la caja de alimentación y los extractores pueden retirarse para que queden accesibles los moldes.

\section{Mecanismos de los moldes superiores}

La segunda leva empuja a un árbol, el cual, a su vez, comunica un movimiento alternativo al molde superior. Cuando alcanza una determinada posición, un dispositivo fiador lo fija quedando en condiciones de resistir el enorme empuje que se aplica a los moldes desde la parte inferior de la prensa.

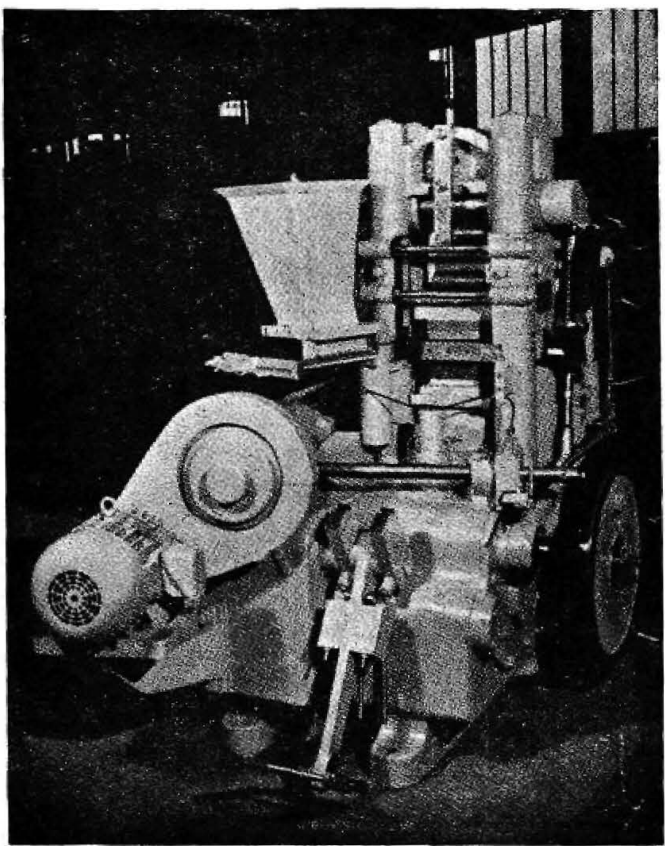

Fig. 5. - Tolva de alimentación retirada lateralmente para hacer accesibles los moldes

Para que el troquel entre perfectamente en la caja de moldeo hay dispuestas unas guías en las columnas del cuerpo central de la prensa (fig. 6). Dichas guías están ampliamente separadas para que la estabilidad sea mejor. En caso de desajuste de las guías por desgaste pueden colocarse unos suplementos previstos con tal fin.

En la figura 6 también pueden verse los enchufes para los calentadores del molde superior, que tienen por finalidad evitar que las tortas de arcilla queden adheridas al molde. El molde inferior también está provisto de calefacción. Una lámpara piloto, montada en el cabezal de la prensa, avisa cuando se encuentra en condiciones de funcionar. Con este sistema de calentadores pueden conseguirse fácilmente temperaturas de $100^{\circ} \mathrm{C}$.

\section{Prensado}

La operación de prensar el azulejo se realiza mediante tres compresiones, producidas a intervalos regulares, que recibe el 


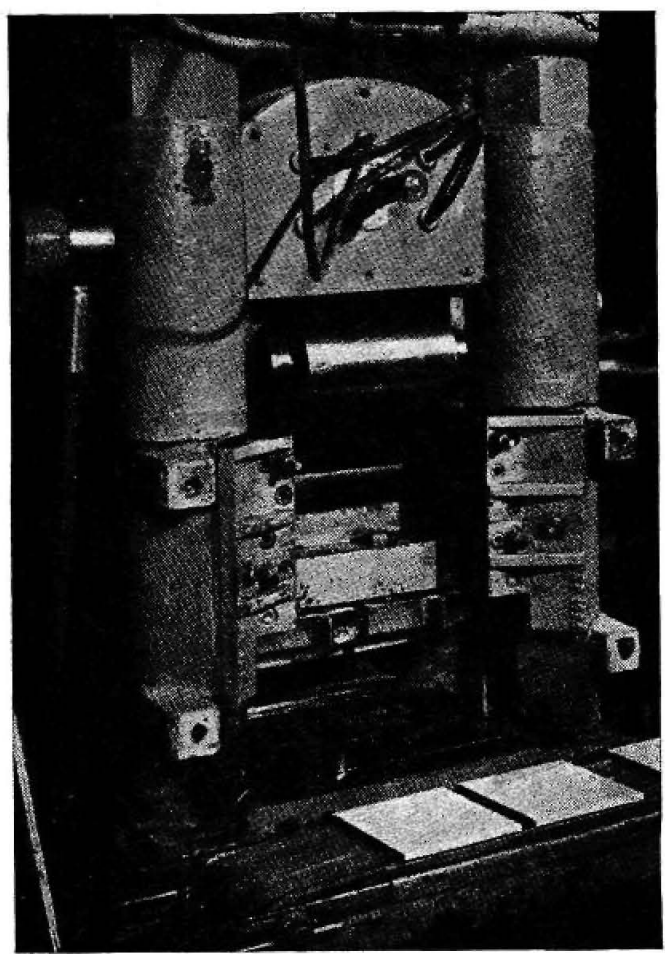

Fig. 6.-Guias para el molde superior, calentadores y lámpara indicadora

troquel inferior por la acción de un juego de mecanismos, los cuales se mueven por medio de sus dos levas correspondientes y utilizando la energía cinética de un volante. Un dispositivo especial oscilante acelera a dicho volante sucesivamente en sentidos de giro diferentes.

\section{Mecanismo extractor}

La última operación realizada mecánicamente es la de retirar las piezas una vez moldeadas. El extractor aplica una compresión inicial a la arcilla, antes de que tenga lugar la compactación, después mantiene a la pieza en su posición adecuada durante el prensado, y, finalmente, realiza la expulsión del azulejo de la caja de moldeo al elevarse el molde inferior a la altura del borde superior de la caja de moldeo.

La fuerza para realizar estos movimientos procede de un juego de resortes, y el ritmo lo gobierna una leva. La compresión inicial sirve para quitar el aire a la pasta antes de realizar la operación de prensado.

\section{Moldes y cajas de moldeo}

La caja de moldeo es de un acero con bajo contenido en carbono y puede ser de una o dos cavidades, según el número de azulejos que se deban prensar simultáneamente. Las superficies interiores están revestidas con camisas de acero, cuya dureza es de 55 a 60 Rockwell C. Los troqueles superior e inferior son también de acero. El paralelismo y verticalidad de los lados se mantienen con una tolerancia de 1,5 milésimas de pulgada y la holgura entre pistones y camisas se calcula con una tolerancia de una milésima por pulgada del tamaño del azulejo.

En esta máquina para variar el tamaño - tipo del azulejo es preciso cambiar los pistones y cajas de moldeo, así como la caja de alimentación $y$, en algunos casos, incluso la tolva alimentadora. Todas estas partes, susceptibles de alteración, son fácilmente accesibles y pueden cambiarse con gran rapidez.

\section{Lubrificación}

La mayoría de las partes móviles de la prensa son lubrificadas a presión por medio de una bomba sumergida, situada en la parte baja de la máquina. Las levas se lubrifican por barboteo en baño de aceite. En aquellos puntos donde es conveniente el engrase a presión se han dispuesto engrasadores de tipo corriente.

\section{Azulejos de canto romo}

El tipo de molde más corriente es el que puede verse en el esquema de la fig. 7. El

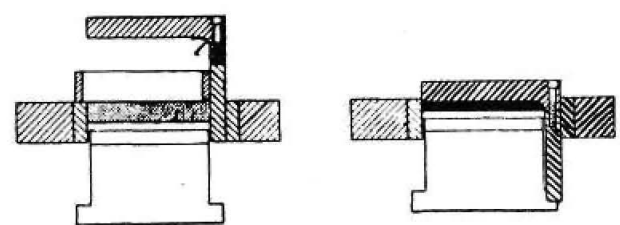

Fig. 7.- Moldes, abierto y cerrado, para fabricar azulejo de canto romo 
borde curvo del azulejo se moldea por la parte abierta de la caja de moldeo, bajando el pistón y dándole la forma durante la operación de prensado.

Es conveniente engrasar la media caña del molde superior para evitar que se pegue la pasta, pudiéndose hacer esto por aspersión o lavado con aceite. Cuando el troquel superior se encuentra en el punto más alto de su recorrido, un sistema de inyectores aspersiona el lubricante. El lavado se realiza por medio de un cepillo montado en la caja alimentadora.

Es raro que estas prensas lleguen a funcionar defectuosamente, pero variando las condiciones de lo arcilla y con cambios en el tipo de azulejo, es posible que ocurro. Con una prensa totalmente mecanizada y automática la corrección de estos errores resulta fácil, una vez que ha sido reconocida la cousa.

\section{Defectos de los azulejos. Piexas blandas}

Cuando los azulejos producidos resultan blandos es muy posible que no se esté utilizando la máxima energía de compre. sión de la máquina. Esto puede ser debido a falta de compactación de la arcilla en el molde, o a defectos en el embrague del eje del volante.

Cuando las arcillos son muy comprensibles se obtienen normalmente azulejos blandos, pudiéndose corregir ésto dando una mayor excentricidad a la leva del pistón compresor. Si se observan zonas blandas en el azulejo hay que reajustar el funcionamiento de la caja alimentadora. Reduciendo la dosificación de agua se pueden obtener piezas más blandas, y, por el contrario, aumentándola se consigue mayor dureza. Como norma general puede establecerse que el contenido de agua debe mantenerse entre un 5 y un $8 \%$, aunque determinadas arcillas precisan cifras que se salen de estos porcentajes.

\section{Estratificaciones o «sopladuras»}

Existe una relación directa entre la presión aplicada y la cantidad de sopladuras que presenta un azulejo. Los mayores por- centajes de estratificaciones se producen cuando los materiales están demasiado secos o demasiado húmedos. Si se aplican presiones muy elevadas pueden mantenerse las pastas muy secas, observándose pocas sopladuras. Por consiguiente, con una presión fija de aproximadamente 40 toneladas la dosificoción óptima de agua, para que el número de azulejos con sopladuras sea muy reducido, estará comprendido entre un 5 y un $8 \%$ si bien esta regla general presenta excepciones. Es esencial mantener la dosificación de agua dentro de este margen, excepto cuando se trata de arcillas con características poco corrientes.

También pueden producirse sopladuras, bien porque la caja de alimentación no es la adecuada para el tipo de azulejo que se está produciendo, y como consecuencia de ello queda insuficientemente relleno el molde, o bien porque la arcilla tiene tendencia a formar bolas y éstas arrastran aire.

Para que no se produzcan estratificaciones es preciso que la holgura entre el pistón y la caja de moldeo sea la estrictamente necesaria. Si es demasiado pequeña, el aire saldrá con dificultad. Un $0,5 \%$ es la holgura óptima. Como los azulejos suelen ser de $10 \mathrm{~cm}$ de lado, la separación será en este caso de $0,5 \mathrm{~mm}$. Según el tipo de pasta utilizada podrá variarse esta dimensión, pero, sin embargo, debe ser la indicada en cada caso para que las partículas de arcilla no tiendan a atascarse entre el pistón y la caja de moldeo, produciendo adherencias y desgastes.

\section{Azulejos deformados}

Las deformaciones que se observan durante la fabricación de los azulejos se deben, principalmente, en unos casos, a falta de uniformidad en el relleno de los moldes, $y$, en otros, a que el mecanismo extractor no realiza su función correctamente. También puede ser debido a que el troquel inferior al descender demasiado de prisa succione algo de pasta.

Las arcillas trituradas con molinos de martillos pueden presentar una granulometría tan irregular que den lugar a pastas que se compacten desigualmente. 
borde curvo del azulejo se moldea por la parte abierta de la caja de moldeo, bajando el pistón y dándole la forma durante la operación de prensado.

Es conveniente engrasar la media caña del molde superior para evitar que se pegue la pasta, pudiéndose hacer esto por aspersión o lavado con aceite. Cuando el troquel superior se encuentra en el punto más alto de su recorrido, un sistema de inyectores aspersiona el lubricante. El lavado se realiza por medio de un cepillo montado en la caja alimentadora.

Es raro que estas prensas lleguen a funcionar defectuosamente, pero variando las condiciones de lo arcilla y con cambios en el tipo de azulejo, es posible que ocurro. Con una prensa totalmente mecanizada y automática la corrección de estos errores resulta fácil, una vez que ha sido reconocida la cousa.

\section{Defectos de los azulejos. Piexas blandas}

Cuando los azulejos producidos resultan blandos es muy posible que no se esté utilizando la máxima energía de compre. sión de la máquina. Esto puede ser debido a falta de compactación de la arcilla en el molde, o a defectos en el embrague del eje del volante.

Cuando las arcillos son muy comprensibles se obtienen normalmente azulejos blandos, pudiéndose corregir ésto dando una mayor excentricidad a la leva del pistón compresor. Si se observan zonas blandas en el azulejo hay que reajustar el funcionamiento de la caja alimentadora. Reduciendo la dosificación de agua se pueden obtener piezas más blandas, y, por el contrario, aumentándola se consigue mayor dureza. Como norma general puede establecerse que el contenido de agua debe mantenerse entre un 5 y un $8 \%$, aunque determinadas arcillas precisan cifras que se salen de estos porcentajes.

\section{Estratificaciones o «sopladuras»}

Existe una relación directa entre la presión aplicada y la cantidad de sopladuras que presenta un azulejo. Los mayores por- centajes de estratificaciones se producen cuando los materiales están demasiado secos o demasiado húmedos. Si se aplican presiones muy elevadas pueden mantenerse las pastas muy secas, observándose pocas sopladuras. Por consiguiente, con una presión fija de aproximadamente 40 toneladas la dosificoción óptima de agua, para que el número de azulejos con sopladuras sea muy reducido, estará comprendido entre un 5 y un $8 \%$ si bien esta regla general presenta excepciones. Es esencial mantener la dosificación de agua dentro de este margen, excepto cuando se trata de arcillas con características poco corrientes.

También pueden producirse sopladuras, bien porque la caja de alimentación no es la adecuada para el tipo de azulejo que se está produciendo, y como consecuencia de ello queda insuficientemente relleno el molde, o bien porque la arcilla tiene tendencia a formar bolas y éstas arrastran aire.

Para que no se produzcan estratificaciones es preciso que la holgura entre el pistón y la caja de moldeo sea la estrictamente necesaria. Si es demasiado pequeña, el aire saldrá con dificultad. Un $0,5 \%$ es la holgura óptima. Como los azulejos suelen ser de $10 \mathrm{~cm}$ de lado, la separación será en este caso de $0,5 \mathrm{~mm}$. Según el tipo de pasta utilizada podrá variarse esta dimensión, pero, sin embargo, debe ser la indicada en cada caso para que las partículas de arcilla no tiendan a atascarse entre el pistón y la caja de moldeo, produciendo adherencias y desgastes.

\section{Azulejos deformados}

Las deformaciones que se observan durante la fabricación de los azulejos se deben, principalmente, en unos casos, a falta de uniformidad en el relleno de los moldes, $y$, en otros, a que el mecanismo extractor no realiza su función correctamente. También puede ser debido a que el troquel inferior al descender demasiado de prisa succione algo de pasta.

Las arcillas trituradas con molinos de martillos pueden presentar una granulometría tan irregular que den lugar a pastas que se compacten desigualmente. 


\section{Otros defectos}

El mecanismo extractor puede ser el culpable de que se produzcan grietas, horizontales o verticales, en los azulejos, asi como también de que se produzcan ala. beos en las piezas.

Por último también es frecuente que se adhieran las piezas a los moldes de las prensas manuales; ahora bien, en los automáticos ésto es todavía mós frecuente, debido a la rapidez de los movimientos mecánicos. Los procedimientos para evitarlo consisten en la lubrificación o calentamiento de los moldes. También puede recurrirse a un sistema que consiste en añadir a la pasta un lubricante.

C. S. C. 